\title{
ECOCARDIOGRAFIA EM PACIENTES COM APNEIA DO SONO GRAVE COM E SEM PRESSÃO ARTERIAL CONTROLADA: ESTUDO TRANSVERSAL
}

ECHOCARDIOGRAPHY IN PATIENTS WITH SEVERE SLEEP

APNEA WITH AND WITHOUT CONTROLLED BLOOD PRESSURE:

CROSS-SECTIONAL STUDY

\section{Denis Martinez ${ }^{1,2,3}$, Martina Madalena Pedroso', Carolina Caruccio Montanari ${ }^{3}$, Micheli Fagundes ${ }^{1}$, Roberto Pacheco da Silva ${ }^{1}$, Cintia Zappe Fiori ${ }^{1}$}

\section{RESUMO}

Introdução: A apneia obstrutiva do sono (AOS) afeta a anatomia e função do coração. Ocorre hipertensão arterial em metade dos casos de AOS, dificultando atribuir a etiologia dessas alterações separadamente à hipertensão arterial ou à apneia do sono.

Métodos: Estudo transversal de pacientes com índice de apneia-hipopneia maior que 50 eventos por hora. As variáveis ecocardiográficas comparadas em indivíduos com hipertensão arterial controlada e não controlada foram: 1) fração de ejeção, 2) diâmetro da aorta, 3) diâmetro do átrio esquerdo, 4) diâmetro de ventrículo direito, 5) diâmetros do ventrículo esquerdo diastólico e sistólico, 6) percentagem delta, 7) espessura do septo, 8) espessura da parede posterior.

Resultados: Foram incluídos 83 voluntários, 50 com pressão arterial não controlada. Em média, a idade era $47 \pm 9,5$ anos, o índice de massa corporal $34 \pm 5,4 \mathrm{Kg} / \mathrm{m}^{2}$, o índice de apneia-hipopneia $86 \pm 18$ eventos/hora. Sessenta pacientes apresentaram anormalidade no ecocardiograma. A hipertrofia de ventrículo esquerdo foi o achado mais comum, sem diferença de frequência em controles (39\%) e em hipertensos (48\%), seguida por disfunção diastólica em controles (27\%) e em hipertensos (32\%).

Conclusões: Indivíduos com apneia do sono grave e pressão arterial controlada apresentam alterações no ecocardiograma de tipo e frequência semelhantes aos com hipertensão não controlada. Isso sugere que a apneia do sono pode causar dano cardíaco independentemente de hipertensão. Quando não explicáveis por hipertensão arterial, achados como hipertrofia de ventrículo esquerdo podem ser provocados por apneia do sono.

Palavras-chave: Ecocardiografia; apneia do sono; hipertensão

\section{ABSTRACT}

Introduction: Obstructive sleep apnea (OSA) affects the cardiac anatomy and function. Hypertension occurs in half the OSA cases, making it difficult to attribute the cause of these changes separately to arterial hypertension or sleep apnea.

Methods: Prospective cross-sectional study of volunteers with apnea-hypopnea index $>50$ events per hour. The echocardiographic variables were analyzed: 1) ejection fraction, 2) aortic diameter, 3) left atrial diameter, 4) right ventricular diameter, 5) diastolic and systolic diameters of the left ventricle, 6) delta percentage, 7) septum thickness, 8) posterior wall thickness.

Clin Biomed Res. 2015;35(4):217-226

1 Programa de Pós-graduação em Ciências da Saúde: Cardiologia e Ciências Cardiovasculares, Universidade Federal do Rio Grande do Sul (UFRGS). Porto Alegre, RS, Brasil.

2 Serviço de Cardiologia, Hospital de Clínicas de Porto Alegre (HCPA). Porto Alegre, RS, Brasil.

3 Programa de Pós-graduação em Medicina: Ciências Médicas, Universidade Federal do Rio Grande do Sul (UFRGS). Porto Alegre, RS, Brasil.

Autor correspondente:

Denis Martinez

E-mail: dm@ufrgs.br

Serviço de Cardiologia Hospital de Clínicas de Porto Alegre Ramiro Barcelos, 2350.

Porto Alegre, RS, Brasil. 
Results: There were 83 participants, 74 men, 50 with hypertension. The average age was $47 \pm 9.5$ years, body mass index of $34 \pm 5.4 \mathrm{~kg} / \mathrm{m}^{2}$, apnea-hypopnea index of $86 \pm 18$ events/hour, and minimum oxygen saturation of $55 \pm 17 \%$. Left ventricular hypertrophy was the most common echocardiographic abnormality in subjects without hypertension (39\%) and with hypertension (48\%), followed by diastolic dysfunction in subjects with normal blood pressure $(27 \%)$ and with high blood pressure $(32 \%)$. There was no statistically significant difference in echocardiographic characteristics between hypertensive subjects with those with normal pressure.

Conclusions: Individuals with normal blood pressure and severe sleep apnea show abnormalities in the echocardiogram with frequency similar to that observed in patients with high blood pressure. This suggests that sleep apnea can cause ventricular overload independently of hypertension. When not explained by high blood pressure, left ventricular hypertrophy can be caused by sleep apnea.

Keywords: Echocardiography; sleep apnea; hypertension

Apneia obstrutiva do sono ocorre devido ao colapso da faringe induzido pelo sono, causando hipóxia intermitente e despertares repetidos, dois fenômenos ligados às consequências da apneia. O primeiro causa estresse oxidativo, semelhante à isquemia-reperfusão, e o segundo, ativação do simpático ${ }^{1}$. Apneia do sono causa hipertensão arterial sistêmica ${ }^{2,3}$. Além disso, indivíduos com apneia têm risco aumentado de hipertensão pulmonar, acidente vascular cerebral, arritmias, doença arterial coronariana e insuficiência cardíaca ${ }^{4}$. Está bem documentado o aumento da mortalidade cardiovascular ${ }^{5,6}$ por todas essas causas ${ }^{7}$.

A incidência e gravidade das consequências cardiovasculares estão associadas à gravidade da apneia do sono. A gravidade da apneia do sono é avaliada pelo índice de apneia-hipopneia (IAH). Calcula-se o $\mathrm{IAH}$, dividindo o número total de apneias e hipopneias pelo número de horas de sono. A presença de um IAH maior do que 30 eventos por hora indica apneia do sono grave . $^{8}$

Os fatores de risco para as doenças cardiovasculares superpõem-se aos da apneia do sono: sexo masculino, idade avançada e índice de massa corporal (IMC) elevado ${ }^{9}$. Em situações como hipertensão resistente, a prevalência de apneia do sono chega a $80 \%{ }^{10}$, gerando dúvida sobre se as consequências cardiovasculares são mediadas pela apneia do sono ou pela hipertensão arterial. A cada apneia, ocorre aumento transitório da pressão arterial tanto sistêmica ${ }^{11}$, quanto pulmonar ${ }^{4}$. Esse aumento de pressão arterial envolve mecanismos de ativação do simpático pelo despertar, de hipóxia intermitente e de oscilações de pressão intratorácica ${ }^{12}$.

Em normotensos com apneia moderada e grave $(\mathrm{IAH} \geq 15)$, Aslan e colaboradore ${ }^{13}$ observaram septo interventricular de $10.5 \mathrm{~mm}$ e diâmetro do átrio esquerdo de $35.6 \mathrm{~mm}$, significantemente maiores do que em indivíduos com apneia leve $(\mathrm{IAH}<15)$, com septo interventricular de $9.5 \mathrm{~mm}$ e átrio de $33.8 \mathrm{~mm}$.

Com base nessa revisão, hipotetizou-se que, na apneia do sono grave, os indivíduos apresentem alterações ecocardiográficas, independentemente do controle da pressão arterial. Investigou-se, por meio de ecocardiografia, as anormalidades cardíacas anatômicas e funcionais em pacientes com IAH $>50$, um nível inequívoco de gravidade da apneia obstrutiva do sono, comparando resultados entre indivíduos com pressão arterial controlada e não controlada.

\section{MÉTODOS}

Foram avaliados indivíduos que realizaram polissonografia de noite inteira em clínica do sono afiliada à universidade. Os critérios de inclusão foram: 1) idade entre 18 e 70 anos, 2) registro de pelo menos 6 horas de sono, e 3) IAH maior do que 50 eventos/hora. Os critérios de exclusão foram: 1) diagnóstico de cardiopatia isquêmica e miocardiopatia, 2) dados incompletos, e 3) impossibilidade de participação. Os indivíduos completaram questionários padronizados que abrangeram dados demográficos, clínicos e sociais.

A polissonografia foi realizada utilizando método padrão conforme descrito anteriormente ${ }^{14}$. Em resumo, foram registrados eletroencefalograma, eletro-oculograma, eletromiograma, eletrocardiograma, fluxo aéreo, esforço respiratório torácico e abdominal, e saturação de oxigênio no sangue arterial. Foram incluídos exames com ao menos seis horas de registro sem artefatos. Os eventos de apneia e hipopneia deveriam ter duração mínima de 10 segundos. Apneia foi registrada quando o fluxo aéreo caísse abaixo de $10 \%$ do basal e hipopneia quando o fluxo caísse abaixo de $50 \%$ do basal, associado à dessaturação de ao menos $3 \%$ ou a despertar. 
A pressão arterial foi medida com esfigmomanômetro convencional pela técnica padrão ${ }^{15}$ na consulta médica e à noite na chegada ao laboratório do sono para realizar polissonografia. A média dessas duas medidas foi usada como critério para definir os grupos do estudo. Os indivíduos com pressão sistólica igual ou superior a $140 \mathrm{mmHg}$ e/ ou diastólica igual ou superior a $90 \mathrm{mmHg}$ foram considerados hipertensos para fins deste estudo. Os demais, normotensos ou hipertensos controlados com medicação, constituíram o grupo controle.

Os indivíduos realizaram ecocardiograma com Doppler bidimensional a cores, medindo: fração de ejeção (FE); diâmetro da aorta (AO); diâmetro do átrio esquerdo $(\mathrm{AE})$; ventrículo direito (VD); espessura ventrículo esquerdo durante a diástole (VEd); espessura ventrículo esquerdo durante a sístole (VEs); percentagem do delta; espessura do septo (ST); espessura da parede posterior (PP). O ecocardiograma foi realizado por cardiologista especialista em ecocardiografia, sem conhecimento do nível de pressão arterial ou do índice de apneia-hipopneia dos pacientes, de acordo com as normas da Sociedade Americana de Ecocardiografia ${ }^{16}$. Hipertrofia de VE foi definida por medidas de parede septal e posterior no corte paraesternal, eixo longo. Os valores encontrados foram considerados como normais ou anormais, de acordo os limites descritos previamente na literatura ${ }^{17}$. Os participantes assinaram o termo consentindo com o uso anônimo dos seus dados, em consonância à Declaração de Helsinki.

Os dados foram analisados usando o software SPSS v.18 (SPSS, Chicago, IL). As diferenças entre os grupos controle e hipertenso foram analisadas pelo teste qui-quadrado e teste $T$ de Student nas análises univariadas. O modelo para prever cada uma das anormalidades ecocardiográficas a partir do grupo (pressão não controlada ou controle) foi ajustado por regressão binária logística para os seguintes regressores: idade maior que a mediana, $\mathrm{IAH}$ maior que a mediana de eventos por hora e obesidade $\left(I M C \geq 30 \mathrm{~kg} / \mathrm{m}^{2}\right)$. Os achados com probabilidade bicaudal de erro alfa menor que $5 \%$ foram considerados significativos.

\section{RESULTADOS}

A Figura 1 mostra o processo de amostragem. De 1985 indivíduos potencialmente elegíveis avaliados em clínica do sono, 1445 foram excluídos por critérios polissonográficos e de idade. Dos 540 restantes, 457 foram excluídos por falta de dados nas avaliações, doença cardíaca e outros motivos. Características clínicas e resultados polissonográficos da amostra estudada estão apresentados na Tabela 1. Do total de 83 incluídos, 53 apresentavam pressão sistólica maior do que $140 \mathrm{mmHg}$ e/ou diastólica maior do que $90 \mathrm{mmHg}$. Quando comparados com indivíduos

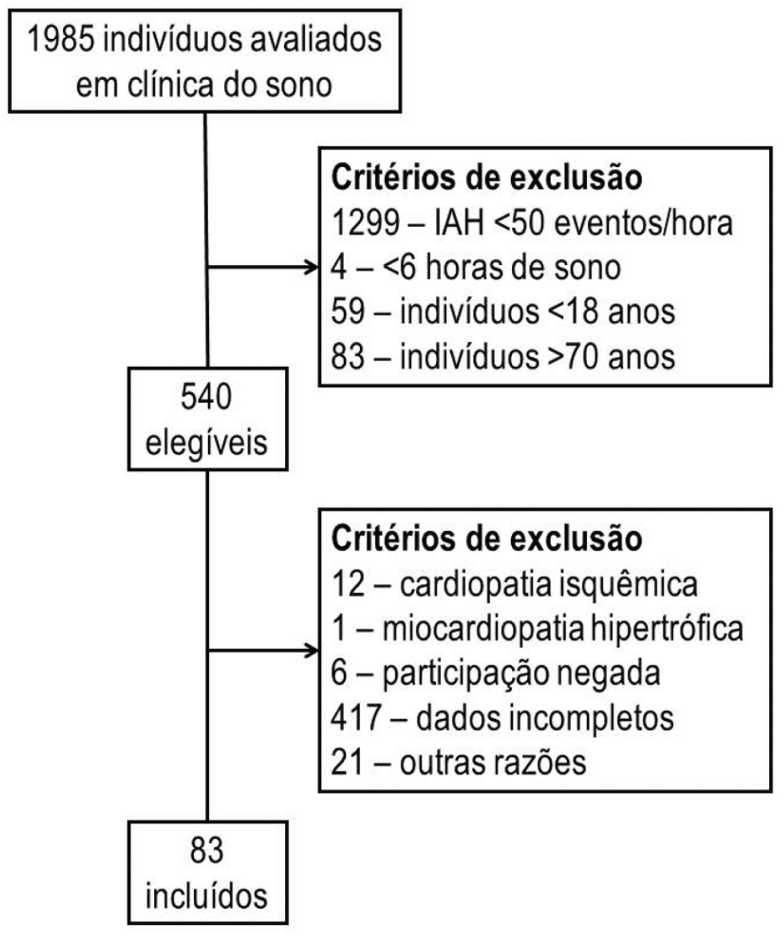

Figura 1: Fluxograma dos participantes.

Tabela 1: Características clínicas e resultados polissonográficos da amostra.

\begin{tabular}{lcccr}
\hline \multicolumn{1}{c}{ Variáveis } & $\begin{array}{c}\text { Total } \\
(\mathbf{n}=\mathbf{8 3})\end{array}$ & $\begin{array}{c}\text { Controles } \\
(\mathbf{n}=\mathbf{3 3})\end{array}$ & $\begin{array}{c}\text { Hipertensos } \\
(\mathbf{n}=\mathbf{5 0})\end{array}$ & P \\
\hline Idade (anos) & $47 \pm 9,5$ & $45 \pm 8,3$ & $49 \pm 9,9$ & 0,06 \\
Sexo masculino & $74(89 \%)$ & $31(94 \%)$ & $43(86 \%)$ & 0,31 \\
Índice de massa corporal (Kg/m2) & $34 \pm 5,4$ & $32 \pm 4,7$ & $35 \pm 5,6$ & $<0,01$ \\
Índice de apneia-hipopneia (eventos/h) & $86 \pm 18,2$ & $85 \pm 19,3$ & $87 \pm 17,6$ & 0,58 \\
Saturação de oxigênio mínima (\%) & $55 \pm 17,4$ & $56 \pm 17,7$ & $55 \pm 17,4$ & 0,97 \\
Pressão arterial sistólica (mmHg) & $140 \pm 18,3$ & $124 \pm 10,2$ & $150 \pm 15,3$ & \\
Pressão arterial diastólica $(\mathbf{m m H g})$ & $91 \pm 13,3$ & $79 \pm 6,9$ & $99 \pm 9,6$ &
\end{tabular}


controles, os hipertensos apresentavam maior média de IMC.

A Tabela 2 apresenta as médias das características ecocardiográficas dos grupos. A Figura 2 mostra as percentagens de casos com achados anormais e a razão de chance de ocorrência de cada achado no grupo exposto a pressão não controlada em relação aos controles. Os grupos não apresentaram diferenças de tipo nem frequência em relação às alterações ecocardiográficas.

Por regressão binária logística se ajustou a razão de chance de todos os achados ecocardiográficos para idade, obesidade e IAH, exceto hipertrofia do VD, pelo pequeno número de casos. A Figura 3 mostra a razão de chance dos indivíduos apresentarem cada uma das anormalidades estudadas. Foi encontrada significância estatística apenas para a associação de risco de disfunção diastólica com idade maior que 45 anos e para alteração de átrio esquerdo com obesidade. Em nenhuma das disfunções a pressão não controlada representa um fator de risco significantemente maior que o controle.

\section{DISCUSSÃO}

A percentagem e o tipo de alterações ecocardiográficas observada nesta amostra de indivíduos com apneia do sono grave não difere entre controles e casos com pressão não controlada, independente de idade, obesidade e IAH. Esses resultados corroboram nossa hipótese inicial e adicionam à literatura que descreve a relação da apneia do sono, de qualquer nível de gravidade, com danos cardíacos. Isso tem relevância clínica, pois pode levar à suspeita de apneia quando indivíduos sem hipertensão arterial ou queixas cardiológicas apresentam anormalidades ecográficas que poderiam ser consideradas "falsos positivos".

As alterações ecocardiográficas relatadas na literatura são mais frequentes quanto mais acentuada a apneia ${ }^{18}$. Como no presente estudo só foram incluídos casos com IAH $>50$, havia a expectativa de que a quase totalidade dos incluídos apresentasse hipertrofia ventricular, principalmente no grupo com hipertensão arterial não controlada. Encontrou-se, porém, percentagem relativamente baixa de hipertrofia ventricular medida pela espessura das paredes septal e posterior em comparação com os dados relatados por Cioffi e colaboradores ${ }^{18}$, que encontraram hipertrofia do ventrículo esquerdo em $58 \%$ dos pacientes com apneia moderada/severa.

No presente estudo, $4 \%$ de nossa amostra apresentou hipertrofia de ventrículo direito. Guidry et al. ${ }^{19}$, observaram que a espessura do ventrículo direito foi significativamente maior em sujeitos com apneia do sono com média de índice de apneia-hipopneia de $42(0,78 \pm 0,02 \mathrm{~cm})$ do que em sujeitos com baixo índice de dessaturação $(0,68 \pm 0,02)$.

Apneia obstrutiva do sono pode contribuir para hipertrofia ventricular pela hipoxemia crônica, exarcebação de hipertensão noturna ${ }^{20}$, hiperatividade do simpático ${ }^{21}$ e redução de tônus do parassimpático ${ }^{22}$. A fisiopatologia inclui: 1) hipóxia desencadeando isquemia e prejuízo da contratilidade, 2) dano aos miócitos por aumento da concentração das catecolaminas circulantes, 3 ) mudança nas pressões intratorácicas alterando o relaxamento e os volumes sistólico e diastólico finais do ventrículo esquerdo ${ }^{23}$.

Os esforços respiratórios durante episódios de apneia obstrutiva geram pressão negativa intratorácica, aumentam a pressão transmural e a pós-carga ventricular. Além disso, o aumento do retorno venoso às câmaras direitas pode resultar em um deslocamento para a esquerda do septo interventricular. Além de dificultar o enchimento do VE, o desvio do septo reduz a pré-carga do VE e o volume sistólico. A diminuição da pressão parcial de $\mathrm{O}_{2}$ e o aumento transitório na pressão parcial de $\mathrm{CO}_{2}$ durante os episódios de apneia estimulam os quimiorreceptores periféricos e centrais, levando à vasoconstrição, elevação da resistência periférica e frequência cardíaca ${ }^{22}$.

Tabela 2: Características ecocardiográficas.

\begin{tabular}{llllc}
\hline \multicolumn{1}{c}{ Variáveis } & Total & Controles & Hipertensos & P \\
\hline Fração de Ejeção (\%) & $69,5 \pm 10,6$ & $70,0 \pm 11,3$ & $69,3 \pm 10,2$ & 0,64 \\
Aorta (mm) & $33,3 \pm 5,4$ & $32,5 \pm 5,4$ & $33,9 \pm 5,4$ & 0,31 \\
Átrio esquerdo (mm) & $35,2 \pm 6,5$ & $34,0 \pm 7,3$ & $36,0 \pm 6,0$ & 0,23 \\
Ventrículo direito (mm) & $21,0 \pm 3,6$ & $21,0 \pm 3,6$ & $21,0 \pm 3,7$ & 0,80 \\
Ventrículo esquerdo em diástole (mm) & $49,6 \pm 6,2$ & $49,3 \pm 5,4$ & $49,8 \pm 6,7$ & 0,22 \\
Ventrículo esquerdo em sístole (mm) & $31,4 \pm 6,6$ & $31,1 \pm 6,6$ & $31,7 \pm 6,6$ & 0,96 \\
\% delta & $36,7 \pm 7,6$ & $37,4 \pm 8,8$ & $36,3 \pm 6,8$ & 0,25 \\
Septo interventricular (mm) & $10,2 \pm 1,6$ & $10,1 \pm 1,6$ & $10,3 \pm 1,6$ & 0,80 \\
Parede posterior (mm) & $10,2 \pm 1,8$ & $10,0 \pm 1,5$ & $10,3 \pm 2,0$ & 0,40 \\
\hline
\end{tabular}



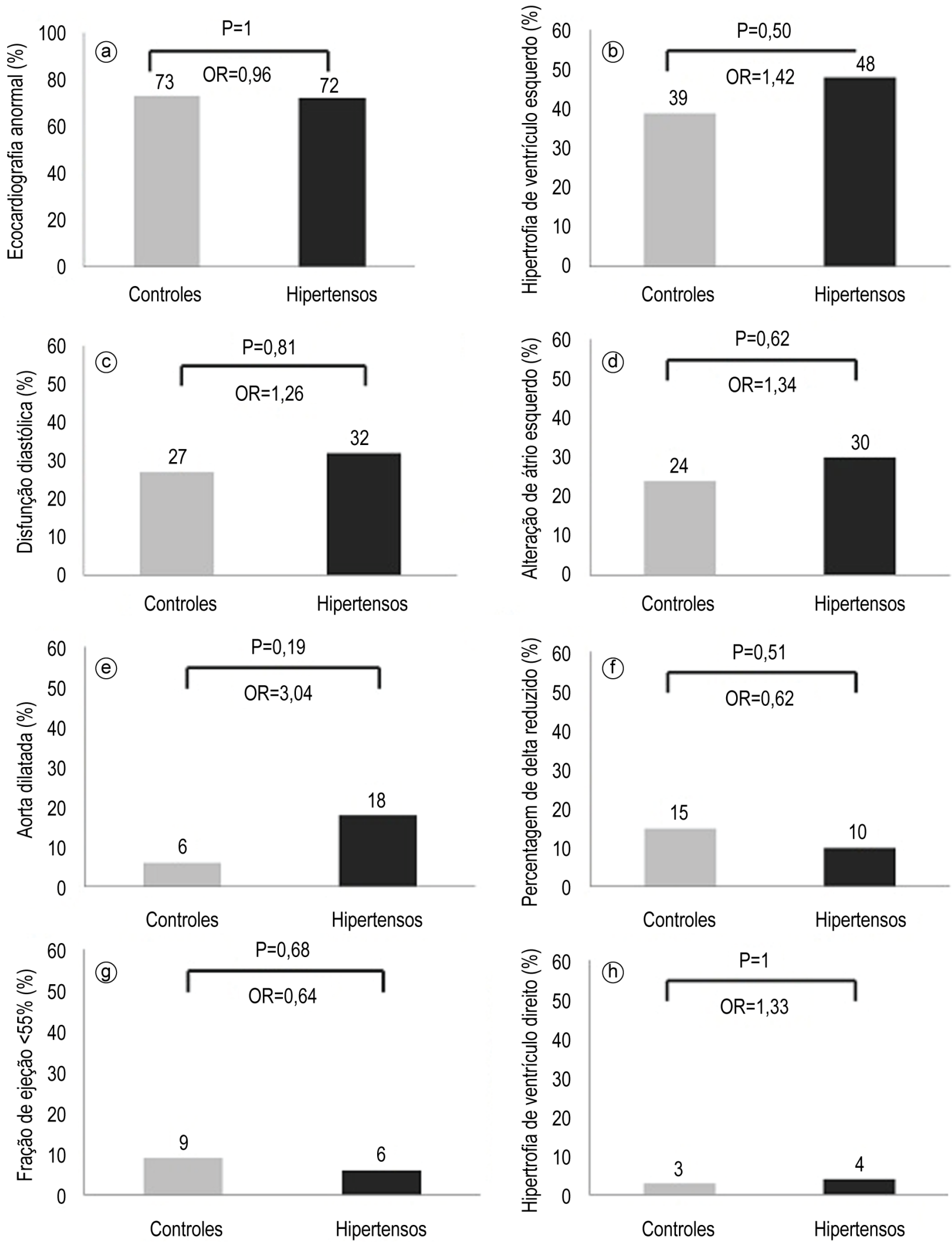

Figura 2: Frequência das alterações ecocardiográficas em indivíduos controles e hipertensos. (a) Percentagem de Ecografia Anormal; (b) Percentagem de Disfunção Diastólica; (c) Percentagem de Aorta Dilatada; (d) Percentagem de Alteração do Átrio Esquerdo; (e) Percentagem de Hipertrofia de Ventrículo Direito; (f) Percentagem de Hipertrofia de Ventrículo Esquerdo; (g) Percentagem de Fração de Ejeção Menor que 55\%; (h) Percentagem de delta reduzido. 


\section{A. Ecocardiograma anormal}

Variável OR $(95 \%$ IC)

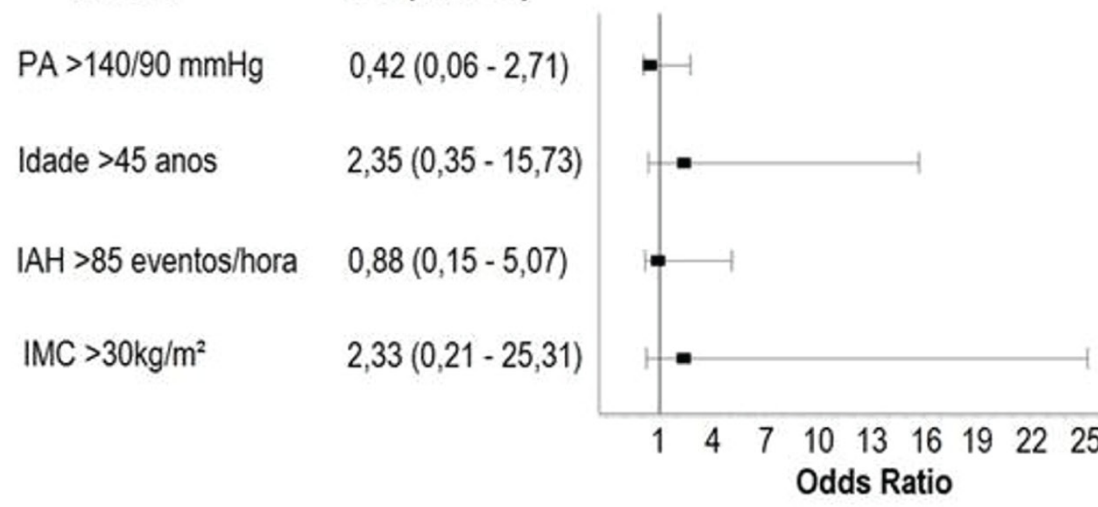

B. Hipertrofia de ventrículo esquerdo

Variável

$\mathrm{PA}>140 / 90 \mathrm{mmHg}$

Idade $>45$ anos

IAH $>85$ eventos/hora

IMC $>30 \mathrm{~kg} / \mathrm{m}^{2}$

$2,43(0,78-7,61)$

$1,03(0,39 \cdot 2,76)$

$1,75(0,68 \cdot 4,5)$

$0,46(0,18-1,18)$

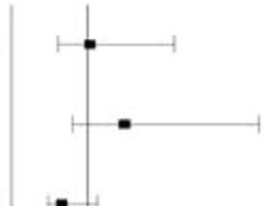

)

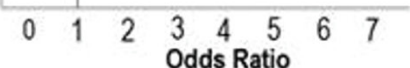

\section{Alteração de átrio esquerdo}

Variável

$\mathrm{PA}>140 / 90 \mathrm{mmHg}$

Idade $>45$ anos

$\mid \mathrm{AH}>85$ eventos/hora

IMC $>30 \mathrm{~kg} / \mathrm{m}^{2}$

\section{OR $(95 \%$ IC)}

$0,91(0,3-2,8)$

$1,47(0,5-4,33)$

$0,71(0,26-1,98)$

$5,57(1,1-28,21)$

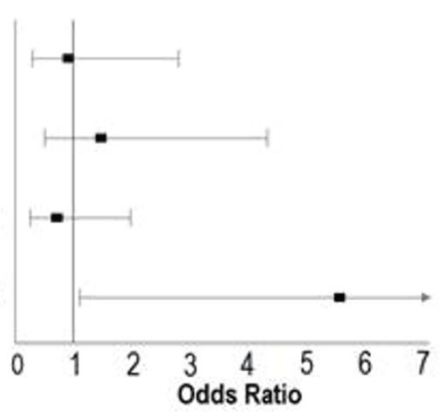

\section{F. Percentagem de delta reduzido}

\section{Variável}

OR $(95 \%$ IC)

$\mathrm{PA}>140 / 90 \mathrm{mmHg}$

$0,41(0,09-1,95)$

Idade $>45$ anos

$1,75(0,38-8,04)$

IAH > 85 eventos/hora $0,33(0,07-1,49)$

IMC $>30 \mathrm{~kg} / \mathrm{m}^{2}$

$2,46(0,41-14,65)$

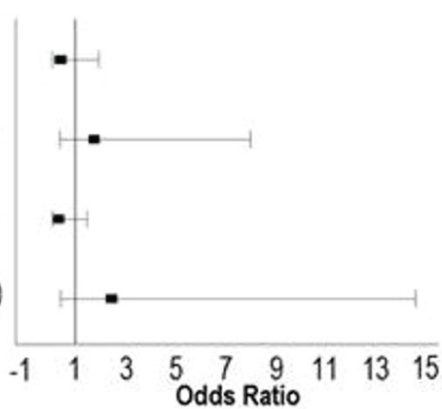

\section{Disfunção diastólica}

OR $(95 \%$ IC)

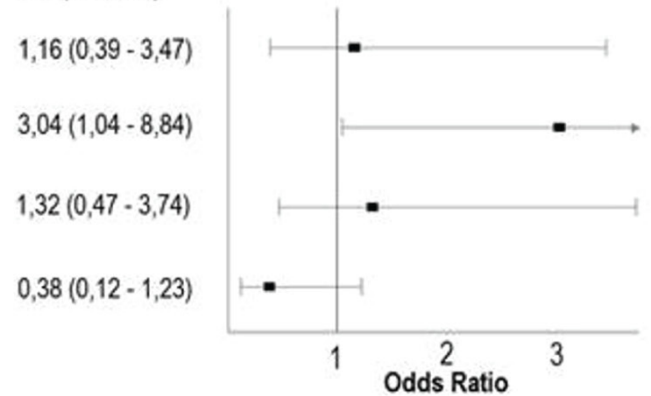

E. Aorta dilatada

OR $(95 \%$ IC)

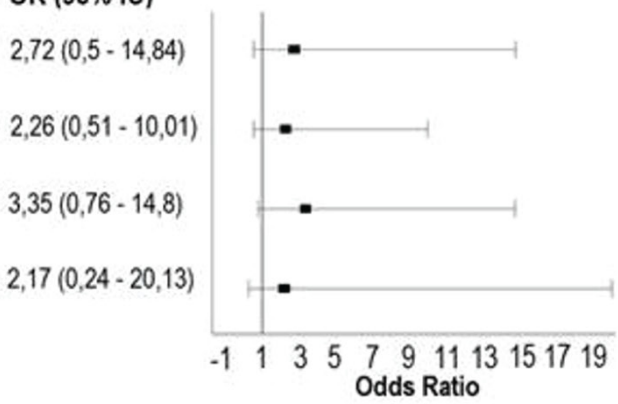

G. Fração de ejeção $<55 \%$

OR $(95 \%$ IC)

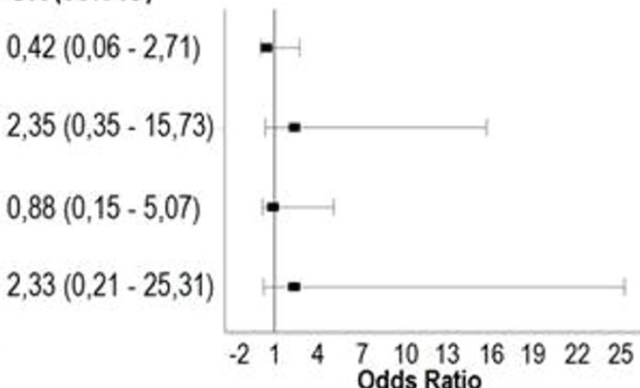

Figura 3: Odds ratio dos pacientes para apresentarem alterações ecocardiográficas: (A) ecocardiografia anormal; (B) disfunção diastólica; (C) aorta dilatada; (D) alteração de átrio esquerdo; (E) hipertrofia de ventrículo esquerda;

(F) fração de ejeção<55\%; (G) Percentagem de delta reduzido. 
Está bem estabelecida a relação da idade com disfunção diastólica ${ }^{24}$. A única variável significante no modelo para prever essa anormalidade, no presente estudo, foi idade acima de 45 anos. Glantze colaboradore ${ }^{25}$ mostraram que disfunção diastólica é mais comum em pacientes com apneia do sono (54\%) do que nos sem apneia $(41 \% ; p=0,02)$. Na análise multivariada, a apneia do sono foi associada com risco 90\% maior de disfunção diastólica. Apesar de os autores não separarem os resultados em relação à presença de hipertensão, nota-se que, entre os casos com apneia do sono, $48 \%$ tinham pressão não controlada e 54\% tinham disfunção diastólica. Portanto, pelo menos uma parte dos casos $(6 \%)$ - provavelmente os mais graves - tinha disfunção diastólica sem pressão elevada. A percentagem de disfunção diastólica nesse estudo é mais alta do que em nossa amostra. Causas possíveis para essa discrepância incluem a exclusão de casos como os deles, com doença coronariana, a menor idade de nossa amostra (64 anos vs. 49 anos) e o método deles para detecção de disfunção diastólica incluir velocidade tecidual e a relação E/e'.

Diversos mecanismos podem explicar a relação independente entre apneia e disfunção diastólica, como a existência de hipertrofia concêntrica, resistência à insulina, ativação neuro-hormonal, aumento do estresse oxidativo e inflamação sistêmica ${ }^{26,27}$. A apneia do sono também pode contribuir para a disfunção diastólica por meio de prejuízo do relaxamento, fibrose e remodelamento vascular ${ }^{28}$.

O relaxamento ventricular envolve gasto energético quando a proteína gigante titina se contrai no início da diástole. Assim, as baixas saturações de oxigênio observadas durante as apneias podem dificultar essa contração e produzir agudamente insuficiência diastólica. Em longo prazo, aumento da rigidez do VE por alterações no colágeno, hipertrofia de miócitos, mudanças na estrutura da matriz extracelular e fibrose intersticial levam à disfunção diastólica ${ }^{29,30}$.

A apneia do sono é a causa mais comum de hipertensão secundária ${ }^{31}$. Há relação dose-resposta entre IAH e prevalência de hipertensão arteria| ${ }^{32}$. Diversas metanálises comprovam que tratar apneia do sono reduz a pressão arterial ${ }^{33,34}$, permitindo que se considere haver relação causa-efeito. A apneia causa alterações estruturais no ventrículo direito $(V D)^{19}$ e no ventrículo esquerdo (VE) ${ }^{35}$. Hipertrofia de $V^{36}$ e insuficiência cardíaca ${ }^{37}$ podem, porém, ocorrer independentemente de apneia do sono, apenas por cardiopatia hipertensiva. Glantz e colaboradores ${ }^{25}$, em 2015, comprovaram que apneia do sono aumenta a chance de disfunção diastólica após revascularização miocárdica.

A função vascular arterial colabora para as anormalidades diastólicas. Alterações na parede vascular interferem com a pós-carga e com a propagação das ondas na parede arterial, aumentando a impedância do VE, ocasionando alentecimento do relaxamento e hipertrofia miocárdica ${ }^{38}$.

É comum o uso do ecocardiograma para avaliar pacientes hipertensos, diabéticos e portadores de doença arterial coronariana. O ecocardiograma é um procedimento pouco utilizado em pacientes assintomáticos. Entretanto, na presença de apneia do sono, mesmo que assintomático do ponto de vista cardíaco, o indivíduo poderá apresentar anormalidades cardíacas. Nestes casos, realizar um questionário simples como o STOP-Bang ${ }^{39}$ pode indicar o risco de apneia do sono e, portanto, indicar avaliação por ecocardiograma.

O STOP-Bang avalia presença de S (snoring), ronco ouvido através da porta fechada; $T$ (tiredness), cansaço frequente; $\mathrm{O}$ (observed), apneias relatadas por observador; P (pressure), pressão arterial elevada; $\mathrm{B}$ (body mass index), índice de massa corporal $>35 \mathrm{~kg} / \mathrm{m} 2$; A (age), idade > 50 anos; $\mathrm{N}$ (neck), colarinho > $40 \mathrm{~cm}$; e G (gender), sexo masculino. Três respostas afirmativas a essas perguntas indicam risco para apneia do sono.

Os achados desse estudo aceitam a hipótese nula, mas são limitados pelo baixo poder estatístico $(24 \%)$. Sendo as diferenças entre os grupos de aproximadamente 0,1 desvio padrão, em termos de achados ecocardiográficos (Tabela 2), seriam necessários mais de mil pacientes em cada grupo para confirmar com poder de $80 \%$ a inexistência de diferença entre grupos com pressão controlada e não controlada, na apneia do sono grave. Inicialmente o estudo foi projetado para encontrar diferenças de 0,5 desvio padrão entre os grupos. Considera-se um tamanho de efeito com o d de Cohen menor que 0,2 como pouco significante clinicamente ${ }^{40}$. Devido ao pequeno tamanho de efeito observado, é improvável que medidas ecocardiográficas mais sofisticadas que as aqui empregadas sejam capazes de detectar diferenças significantes.

O desenho do estudo não incluiu controles sem apneia do sono. Como se esperava encontrar anormalidades na quase totalidade dos indivíduos, considerou-se que a comparação com indivíduos normais não acrescentaria conhecimento sobre a questão de pesquisa, i.e., o papel relativo da pressão não controlada e da apneia do sono.

A diferença entre o número de homens e mulheres não permitiu explorar o efeito do sexo nas anormalidades ecocardiográficas. Essa disparidade era esperada pela prevalência de apneia na população geral ser maior em homens do que em mulheres ${ }^{41}$, mas a desproporção foi maior que o previsto, provavelmente por serem casos extremamente graves. 
Ocorreu grande perda de casos em relação ao total elegível, limitando a validade externa do estudo. Por ser esta amostra derivada de pacientes com queixas de sono, não de pacientes cardiológicos, a indicação de realizar ecocardiografia produz viés de seleção. Os dados têm validade interna, mas não servem, de forma alguma, como evidência epidemiológica por falta de validade externa. Os números obtidos são percentagens e não expressam prevalência.

Apesar de este estudo permitir que sejam geradas hipóteses, para esclarecer definitivamente o papel da coexistência de apneia do sono e hipertensão arterial sobre a anatomia e função cardíacas, serão necessários estudos incluindo amostra mais numerosa, com representação do sexo feminino e grupos controle com e sem apneia do sono e com e sem pressão controlada. Além disso, pesquisas futuras deveriam incluir técnicas ecodopplercardiográficas como o eco Doppler tecidual e Speckle Tracking.

\section{CONCLUSÃO}

Este estudo contribui para o entendimento do papel da apneia obstrutiva do sono na gênese de anormalidades cardíacas. Os resultados sugerem que, na apneia do sono grave, tanto alterações anatômicas (hipertrofia ventricular) como funcionais (disfunção diastólica) podem ocorrer independente da pressão arterial elevada. Alterações no ecocardiograma, quando não explicáveis por hipertensão arterial, devem provocar suspeita de apneia do sono.

\section{Declaração}

Denis Martinez é proprietário de uma clínica do sono particular. Nada a ser declarado pelos demais autores.

\section{Contribuições dos autores}

Concepção, desenho da pesquisa e obtenção de dados: Martinez D; Análise estatística, interpretação dos dados, redação e revisão crítica do manuscrito quanto ao conteúdo intelectual importante: Martinez D, Pedroso MM, Montanari CC, Fagundes M, da Silva RP e Fiori CZ.

\section{Fonte de financiamento}

O presente estudo não teve fontes de financiamento externas.

\section{REFERÊNCIAS}

1. Dempsey JA, Veasey SC, Morgan BJ, O'Donnell CP. Pathophysiology of sleep apnea. Physiol Rev. 2010;90(1):47-112. http://dx.doi. org/10.1152/physrev.00043.2008. PMid:20086074.

2. Konecny $\mathrm{T}$, Kara $\mathrm{T}$, Somers VK. Obstructive sleep apnea and hypertension: an update. Hypertension. 2014;63(2):2039. http://dx.doi.org/10.1161/ HYPERTENSIONAHA.113.00613. PMid:24379177.

3. Vongpatanasin W. Resistant hypertension: a review of diagnosis and management. JAMA. 2014;311(21):2216-24. http://dx.doi. org/10.1001/jama.2014.5180. PMid:24893089.

4. Somers VK, White DP, Amin R, Abraham WT, Costa F, Culebras $A$, et al. Sleep apnea and cardiovascular disease: an American Heart Association/american College Of Cardiology Foundation Scientific Statement from the American Heart Association Council for High Blood Pressure Research Professional Education Committee, Council on
Clinical Cardiology, Stroke Council, and Council On Cardiovascular Nursing. In collaboration with the National Heart, Lung, and Blood Institute National Center on Sleep Disorders Research (National Institutes of Health). Circulation. 2008;118(10):1080111. http://dx.doi.org/10.1161/ CIRCULATIONAHA.107.189420. PMid:18725495.

5. Martínez-García MA, CamposRodríguez F, Catalán-Serra $P$, SolerCataluña JJ, Almeida-Gonzalez C, De la Cruz Morón I, et al. Cardiovascular mortality in obstructive sleep apnea in the elderly: role of long-term continuous positive airway pressure treatment: a prospective observational study. Am J Respir Crit Care Med. 2012;186(9):909-16. http://dx.doi. org/10.1164/rccm.201203-0448OC. PMid:22983957.

6. Xie W, Zheng F, Song X. Obstructive sleep apnea and serious adverse outcomes in patients with cardiovascular or cerebrovascular disease: a PRISMA-compliant systematic review and metaanalysis. Medicine (Baltimore). 2014;93(29):e336. http://dx.doi. org/10.1097/MD.0000000000000336. PMid:25546682.

7. Marshall NS, Wong KK, Liu PY, Cullen SR, Knuiman MW, Grunstein RR. Sleep apnea as an independent risk factor for all-cause mortality: the Busselton Health Study. Sleep. 2008;31(8):1079-85. PMid:18714779.

8. Sleep-related breathing disorders in adults: recommendations for syndrome definition and measurement techniques in clinical research. The Report of an American Academy of Sleep Medicine Task Force. Sleep. 1999;22(5):667-89. PMid:10450601.

9. Yaggi HK, Strohl KP. Adult obstructive sleep apnea/hypopnea syndrome:definitions, risk factors, and pathogenesis. Clin Chest Med. 2010;31(2):179-86. http://dx.doi. org/10.1016/j.ccm.2010.02.011. PMid:20488280.

10. Gonçalves SC, Martinez D, Gus M, de Abreu-Silva EO, Bertoluci C, Dutra I, et al. Obstructive sleep apnea and resistant hypertension: a case-control study. Chest. 2007;132(6):1858- 
62. http://dx.doi.org/10.1378/ chest.07-1170. PMid:18079220.

11. Kapa S, Sert Kuniyoshi FH, Somers VK. Sleep Apnea and hypertension: interactions and implications for management. Hypertension. 2008;51(3):6058. http://dx.doi.org/10.1161/ HYPERTENSIONAHA.106.076190. PMid:18227409.

12. Dewan NA, Nieto FJ, Somers VK. Intermittent hypoxemia and OSA: implications for comorbidities. Chest. 2015;147(1):266-74. http:// dx.doi.org/10.1378/chest.14-0500. PMid:25560865.

13. Aslan K, Deniz A, Cayli M, Bozdemir H, Sarica Y, Seydaoglu G. Early left ventricular functional alterations in patients with obstructive sleep apnea syndrome. Cardiol J. 2013;20(5):51925. http://dx.doi.org/10.5603/ CJ.2013.0043. PMid:24469876.

14. Martinez D, Breitenbach TC, Lumertz MS, Alcântara DL, Rocha NS, Cassol $\mathrm{CM}$, et al. Repeating administration of Epworth Sleepiness Scale is clinically useful. Sleep Breath. 2011;15(4):76373. http://dx.doi.org/10.1007/s11325010-0434-4. PMid:21063794.

15. Sociedade Brasileira de Cardiologia. IV Diretrizes Brasileiras de hipertensão arterial. Arq Bras Cardiol. 2004;82(Supl 4):7-14. PMid:15107944.

16. Quiñones MA, Otto $C M$, Stoddard M, Waggoner A, Zoghbi WA. Recommendations for quantification of Doppler echocardiography: a report from the Doppler Quantification Task Force of the Nomenclature and Standards Committee of the American Society of Echocardiography. J Am Soc Echocardiogr. 2002;15(2):16784. http://dx.doi.org/10.1067/ mje.2002.120202. PMid:11836492.

17. Schvartzman PR, Fuchs FD, Mello AG, Coli M, Schvartzman M, Moreira LB. Normal values of echocardiographic measurements. A population-based study. Arq Bras Cardiol. 2000;75(2):10714. http://dx.doi.org/10.1590/ S0066-782X2000000800003. PMid:10983027.

18. Cioffi G, Russo TE, Stefenelli C, Selmi A, Furlanello F, Cramariuc $D$, et al. Severe obstructive sleep apnea elicits concentric left ventricular geometry. J Hypertens. 2010;28(5):1074-82. http://dx.doi.
org/10.1097/HJH.0b013e328336c90a. PMid:20411620.

19. Guidry UC, Mendes LA, Evans JC, Levy D, O'Connor GT, Larson $M G$, et al. Echocardiographic features of the right heart in sleep-disordered breathing: the Framingham Heart Study. Am J Respir Crit Care Med. 2001;164(6):933-8. http://dx.doi. org/10.1164/ajrccm.164.6.2001092. PMid:11587973.

20. Turkbey EB, McClelland RL, Kronmal RA, Burke GL, Bild DE, Tracy $R P$, et al. The impact of obesity on the left ventricle: the Multi-Ethnic Study of Atherosclerosis (MESA). JACC Cardiovasc Imaging. 2010;3(3):26674. http://dx.doi.org/10.1016/j. jcmg.2009.10.012. PMid:20223423.

21. Bradley TD, Floras JS. Sleep apnea and heart failure: Part I: obstructive sleep apnea. Circulation. 2003;107(12):16718. http://dx.doi.org/10.1161/01. CIR.0000061757.12581.15. PMid:12668504.

22. Johnson CB, Beanlands RS, Yoshinaga K, Haddad H, Leech J, de Kemp R, et al. Acute and chronic effects of continuous positive airway pressure therapy on left ventricular systolic and diastolic function in patients with obstructive sleep apnea and congestive heart failure. Can J Cardiol. 2008;24(9):697-704. http://dx.doi.org/10.1016/S0828282X(08)70668-8. PMid:18787720.

23. Cintra FD, Poyares D, Guilleminault C, Carvalho AC, Tufik S, de Paola AA. Cardiovascular comorbidities and obstructive sleep apnea. Arq Bras Cardiol. 2006;86(6):399-407. PMid:16810413.

24. Wachter R, Lüthje L, Klemmstein $D$, Lüers C, Stahrenberg R, Edelmann $F$, et al. Impact of obstructive sleep apnoea on diastolic function. Eur Respir J. 2013;41(2):376-83. http://dx.doi. org/10.1183/09031936.00218211. PMid:22790918.

25. Glantz H, Thunström E, Johansson MC, Wallentin Guron C, Uzel H, Ejdebäck J, et al. Obstructive sleep apnea is independently associated with worse diastolic function in coronary artery disease. Sleep Med. 2015;16(1):160-7. http://dx.doi. org/10.1016/j.sleep.2014.08.018. PMid:25547036.

26. Niroumand M, Kuperstein R, Sasson Z, Hanly PJ. Impact of obstructive sleep apnea on left ventricular mass and diastolic function. Am J Respir Crit Care Med. 2001;163(7):1632-6. http://dx.doi. org/10.1164/ajrccm.163.7.2007014. PMid:11401886.

27. Hedner J, Franklin K, Peker Y. Coronary artery disease and sleep apnea. In: Kryger MH, Roth T, Dement WC, editors. Principals and practice of sleep medicine. 5th ed. Philadelphia: Elsevier Inc; 2011. p. 1393-9.

28. Friedrich SP, Lorell BH, Rousseau MF, Hayashida W, Hess OM, Douglas $\mathrm{PS}$, et al. Intracardiac angiotensinconverting enzyme inhibition improves diastolic function in patients with left ventricular hypertrophy due to aortic stenosis. Circulation. 1994;90(6):276171. http://dx.doi.org/10.1161/01. CIR.90.6.2761. PMid:7994819.

29. Borbély A, Falcao-Pires I, van Heerebeek L, Hamdani N, Edes I, Gavina C, et al. Hypophosphorylation of the Stiff N2B titin isoform raises cardiomyocyte resting tension in failing human myocardium. Circ Res. 2009;104(6):7806. http://dx.doi.org/10.1161/ CIRCRESAHA.108.193326. PMid:19179657.

30. Huang B, Wang S, Qin D, Boutjdir M, El-Sherif N. Diminished basal phosphorylation level of phospholamban in the postinfarction remodeled rat ventricle: role of beta-adrenergic pathway, G(i) protein, phosphodiesterase, and phosphatases. Circ Res. 1999;85(9):848-55. http://dx.doi. org/10.1161/01.RES.85.9.848. PMid:10532953.

31. Chobanian AV, Bakris GL, Black HR, Cushman WC, Green LA, Izzo JL $J R$, et al. The seventh report of the joint national committee on prevention, detection, evaluation, and treatment of high blood pressure: the JNC 7 report. JAMA. 2003;289(19):2560-72. http:// dx.doi.org/10.1001/jama.289.19.2560. PMid:12748199.

32. Peppard PE, Young T, Palta M, Skatrud J. Prospective study of the association between sleep-disordered breathing and hypertension. $N$ Engl J Med. 2000;342(19):137884. http://dx.doi.org/10.1056/ NEJM200005113421901. PMid:10805822.

33. Iftikhar IH, Valentine $\mathrm{CW}$, Bittencourt LR, Cohen DL, Fedson AC, Gíslason $T$, et al. Effects of continuous positive airway pressure on blood 
pressure in patients with resistant hypertension and obstructive sleep apnea: a meta-analysis. $J$ Hypertens. 2014;32(12):234150, discussion 2350. http://dx.doi. org/10.1097/HJH.0000000000000372. PMid:25243523.

34. Iftikhar IH, Hays ER, Iverson MA, Magalang UJ, Maas AK. Effect of oral appliances on blood pressure in obstructive sleep apnea: a systematic review and meta-analysis. J Clin Sleep Med. 2013;9(2):165-74. PMid:23372472.

35. Romero-Corral A, Somers VK, Pellikka PA, Olson EJ, Bailey KR, Korinek $\mathrm{J}$, et al. Decreased right and left ventricular myocardial performance in obstructive sleep apnea. Chest. 2007;132(6):1863-70. http:// dx.doi.org/10.1378/chest.07-0966. PMid:17908706.
36. Devereux RB, Roman MJ. Left ventricular hypertrophy in hypertension: stimuli,patterns, and consequences. Hypertens Res. 1999;22(1):1-9. http:// dx.doi.org/10.1291/hypres.22.1. PMid:10221344.

37. Narayanan A, Aurigemma GP, Chinali M, Hill JC, Meyer TE, Tighe DA. Cardiac mechanics in mild hypertensive heart disease: a speckle-strain imaging study. Circ Cardiovasc Imaging. 2009;2(5):38290. http://dx.doi.org/10.1161/ CIRCIMAGING.108.811620. PMid:19808626.

38. Tsujino T, Kawasaki D, Masuyama T. Left ventricular diastolic dysfunction in diabetic patients: pathophysiology and therapeutic implications. Am J Cardiovasc Drugs. 2006;6(4):219-30. http://dx.doi.org/10.2165/00129784200606040-00002. PMid:16913823.

39. Farney RJ, Walker BS, Farney RM, Snow GL, Walker JM. The STOPBang equivalent model and prediction of severity of obstructive sleep apnea: relation to polysomnographic measurements of the apnea/ hypopnea index. J Clin Sleep Med. 2011;7(5):459-65B. PMid:22003340.

40. Cohen J. A power primer. Psychol Bull. 1992;112(1):155-9. http://dx.doi. org/10.1037/0033-2909.112.1.155. PMid:19565683.

41. Tufik S, Santos-Silva R, Taddei JA, Bittencourt LR. Obstructive sleep apnea syndrome in the Sao Paulo epidemiologic sleep study. Sleep Med. 2010;11(5):441-6. http://dx.doi. org/10.1016/j.sleep.2009.10.005. PMid:20362502.

Recebido: Nov 04, 2015 Aceito: Dez 09, 2015 